Log-Concave Probability and Its Applications

by

Mark Bagnoli, Ted Bergstrom

University of Michigan

Current version: October 1989 


\title{
Log-Concave Probability and Its Applications
}

\author{
Mark Bagnoli, Ted Bergstrom
}

\section{Introduction.}

Many interesting propositions in the economics of information are built on the assumption that the log of the cumulative distribution function of a random variable is a concave function. This property is equivalent to the ratio of the density function to the c.d.f. being a monotone decreasing function.

With games of incomplete information, it is customary to convert the game into a game of imperfect, but complete, information by assuming that an opponent of unknown characteristics is drawn from a probability distribution over a set of possible "types" of player. For example, in the literature on contracts, it is assumed that the principal does not know a relevant characteristic of an agent. From the principal's point of view the agent's type is a random variable, with distribution function, $F$. It is standard to assume, as do Laffont and Tirole (1988), that $F$ is log-concave. This assumption is required to make the optimal incentive contract invertible in the agent's type and thus to ensure a separating equilibrium. In the theory of regulation, the regulator does not know the firm's costs. Baron and Myerson (1982) show that a sufficient condition for existence of a separating equilibrium is that the distribution function of types is log-concave. Rob (1989) in a study of pollution claim settlements, Lewis and Sappington (1988) in a study of regulatory theory, and Riordan and Sappington (1989), in a study of government procurement, use essentially the same condition. Log-concavity also arises in the analysis of auctions. Myerson and Satterthwaite (1983), Matthews (1987), and Maskin and Riley (1984), impose conditions that are implied by log-concavity of the distribution function in order to characterize efficient auctions.

Nalebuff and Caplin (1988) develop a theory of spatial economics, in which there is a set of agents described by a density function, $f$. They are able to demonstrate the existence of a pure-strategy equilibrium in prices for a quite general environment if they assume log-concavity or $f$, or a generalization which they call $\rho$-concavity. In a related paper, Nalebuff and Caplin (1989) find powerful applications of $\rho$-concavity to the theory of elections and to the theory of income distribution.

Bagnoli and Khanna (1989) have a model of the real estate market, where there is a distribution of reservation demands for houses. In this model, sellers choose $p$ to maximize $p(1-F(p))$. In this model, logconcavity of the reliability function, $\bar{F}$ is sufficient to guarantee that solutions are unique and well-behaved. The same condition appears in the finance literature in a study of takeovers by Jegadeesh and Chowdry (1989).

Flinn and Heckman (1983) study a model of job search in which job offers arrive as a Poisson process and where the wage associated with a job offer is drawn from a random variable with distribution function $F$. They show that if the function defined by $H(x)=\int_{x}^{\infty}(1-F(t)) d t$ is log-concave, then with optimal search strategies, an increase in the rate of arrivals of job offers will increase the exit rate from unemployment. Flinn and Heckman's condition is equivalent to a condition that is occasionally studied in the operations research literature on reliability theory (e.g. Muth, (1975). If the random variable $\mathbf{X}$ denotes the lifetime of a machine or an organism, then it happens that log-concavity of $H$ is equivalent to the property that expected remaining length of life diminishes as the machine or the organism gets older.

Bagnoli and Bergstrom (1989a) develop a marriage market model in which there is asymmetric information about the quality of persons as potential marriage partners and where quality is revealed with the passage of time. In this model, if quality, appropriately measured, is distributed with a c.d.f., $F(x)$, then there will be a unique equilibrium distribution of marriages by age and quality of the partners if the function defined by $G(x)=\int_{-\infty}^{x} F(t) d t$ is a log-concave function. As we demonstrate in this paper, log-concavity of

We are grateful to Ken Binmore for suggesting a key ingredient for our proof and to Eduardo Ley for some wizardry with Mathematica. 
$G$ also turns out to be the key assumption in establishing uniqueness of equilibrium in a "lemons market" with costly appraisals.

Since the assumption of log-concavity is much used, it would be nice to be know just when it applies. With many familiar probability distributions, there is no simple closed form expression, either for the c.d.f. or for its integral. This makes it very difficult to verify directly whether either the c.d.f. or its integral is a log-concave function. On the other hand, most commonly-used distributions present us with a relatively simple closed-form density function, $f(x)$. For such functions, it a matter of straightforward calculus to determine whether the density function is log-concave.

Fortunately, it happens that a sufficient condition for the c.d.f. to be log-concave is for the density function to be log-concave and a sufficient condition for the integral of the c.d.f to be log-concave is that the c.d.f. is log-concave. These results are proved in a paper by Prèkopa (1972) in a Hungarian mathematics journal. Flinn and Heckman (1983) introduced these results to the economics literature and they are also applied by Caplin and Nalebuff (1988).

Closely related theorems can be found the industrial engineering literature in the context of reliability theory. Barlow and Proschan (1981, pages 76-79) state similar results and sketch a method of proof. As we will show, there are a few other "tricks" that can be used to identify log-concave distribution functions when the density function is not concave.

The object of this paper is to extend and systematize the known results on log-concave densities, distribution functions, reliability functions and their integrals. We also explain the relation between the concepts used in the economics of information to those used in reliability theory. We present a new proof of the inheritance of log-concavity from density functions to distribution functions and from distribution functions to their integrals. This proof is direct and elementary, depending only on the generalized mean value theorem from calculus. Prèkopa's proof, in contrast, derives this result as a consequence of a much more elaborate theory and the proof is accordingly much more difficult. Finally, we present a detailed catalog of common distributions and information about the log-concavity of their density functions, their distribution functions, their reliability functions, and of the integrals of the distribution functions and reliability functions.

\section{Log-concavity Breeds Log-concavity.}

\section{General Results on Log-concavity.}

The results in this section concern the log-concavity of twice-differentiable real-valued functions, $g$, whose domain is an interval on the extended real line. The remarks, lemmas, and theorems in the remainder of this paper implicitly assume these properties.

Definition. A function $g$ is said to be log-concave on the interval $(a, b)$ if the function $\ln g$ is a concave function on $(a, b)$.

A simple application of calculus informs us of the following.

Remark 1. Log-concavity of $g$ on $(a, b)$ is equivalent to each of the following two conditions.

i. $g^{\prime}(x) / g(x)$ is monotone decreasing on $(a, b)$.

ii. $(\ln g(x))^{\prime \prime}<0$.

Lemma 1. Let $g$ be strictly monotonic (increasing or decreasing) defined on the interval $(a, b)$. Suppose that either $g(a)=0$ or $g(b)=0$. Then if $g^{\prime}$ is a log-concave function on $(a, b)$, it must be that $g(x)$ is also a log-concave function on $(a, b)$.

Proof: Suppose that $g(a)=0$. According to the generalized mean value theorem of calculus (sometimes 
called the "Cauchy mean value theorem"), there exists $\xi \in(a, x)$ such that

$$
\frac{g^{\prime}(x)-g^{\prime}(a)}{g(x)-g(a)}=\frac{g^{\prime \prime}(\xi)}{g^{\prime}(\xi)} .
$$

Suppose that $g^{\prime \prime} / g^{\prime}$ is monotone decreasing. Then since $g(a)=0$ and $x>\xi$, it follows from Equation (1) that

$$
\frac{g^{\prime}(x)-g^{\prime}(a)}{g(x)}>\frac{g^{\prime \prime}(x)}{g^{\prime}(x)} .
$$

Since $g$ is strictly monotonic and $g(a)=0$, it must be that $g(x)$ is of the same sign as $g^{\prime}(x)$ for all $x \in$ $(a, b)$. Therefore multiplying both sides of Inequality $(2)$ by $g(x) g^{\prime}(x)$ preserves the direction of inequality. Performing this multiplication, we have $g^{\prime}(x)^{2}-g^{\prime}(x) g^{\prime}(a)>g(x) g^{\prime \prime}(x)$ and hence $g(x) g^{\prime \prime}(x)-g^{\prime}(x)^{2}<$ $-g^{\prime}(x) g^{\prime}(a)<0$. Therefore

$$
0>\frac{g(x) g^{\prime \prime}(x)-g^{\prime}(x)^{2}}{g^{\prime}(x)^{2}}=\left(\frac{g^{\prime}(x)}{g(x)}\right)^{\prime} .
$$

Remark 1 then enables us to conclude that $g$ is log-concave.

Suppose that $g(b)=0$. By the generalized mean value theorem, there exists $\xi \in(x, b)$ such that

$$
\frac{g^{\prime}(x)-g^{\prime}(b)}{g(x)-g(b)}=\frac{g^{\prime \prime}(\xi)}{g^{\prime}(\xi)} .
$$

If $g^{\prime \prime}(x) / g^{\prime}(x)$ is monotone decreasing, then since $g(b)=0$ and and $x<\xi$, it follows from Equation (4) that

$$
\frac{g^{\prime}(x)-g^{\prime}(b)}{g(x)}<\frac{g^{\prime \prime}(x)}{g^{\prime}(x)} .
$$

Since $g(x)$ is monotone and $g(b)=0$, it must be that for $x<b, g^{\prime}(x) g(x)<0$. Multiplying both sides of Inequality (5) by $g(x) g\left(x^{\prime}\right)$, we have $g^{\prime}(x)^{2}-g^{\prime}(x) g^{\prime}(\hat{x})>g(x) g^{\prime \prime}(x)$. As before, this inequality implies Inequality (3), which together with Remark 1 establishes the log-concavity of $g$.

Log-concavity of Density and its Left Side Integrals.

Let $\mathbf{X}$ be a real-valued random variable whose support is an interval $(\ell, h)$ on the extended real line. Let $\mathbf{X}$ have a cumulative distribution function, $F$, and a density function, $f$, where $f(x)=F^{\prime}(x)$. For all $x \in(\ell, h)$, denote the left side integral of the c.d.f. by $G(x)=\int_{\ell}^{x} F(t) d t$. We show that log-concavity of $f$ implies $\log$-concavity of $F$, which in turn implies $\log$-concavity of $G$. We speak of "left side integrals" here because $F(x)$ and $G(x)$ measure areas lying to the left of $x$ on a histogram.

\section{Theorem 1.}

i. If the density function, $f$, is log-concave on $(\ell, h)$, then the cumulative distribution function, $F$, is also log-concave on $(\ell, h)$.

ii. If the cumulative distribution function, $F$ is log-concave on $(\ell, h)$, then the left side integral of the c.d.f, $G$, is also a log-concave function on on $(\ell, h)$.

Proof: To prove Assertion (i), apply Lemma 1 to the function $F$. Since $F$ is a cumulative distribution function with support $[\ell, h], F(\ell)=0$ and $F$ is strictly increasing on $[\ell, h]$. From Lemma 1 , it follows that if $F^{\prime \prime} / F^{\prime}$ is monotone decreasing, then so is $F^{\prime} / F$. But $F^{\prime}=f$ and $F^{\prime \prime}=f^{\prime}$. Therefore if $f^{\prime} / f$ is monotone decreasing, then $F^{\prime} / F$ is monotone decreasing. Assertion (i) is immediate from this fact and Remark 1.

To prove Assertion (ii), we apply Lemma 1 to the function $G(x)$. Evidently $G(\ell)=0$ and $G$ is a strictly increasing function on $[\ell, h]$. Therefore if $G^{\prime \prime} / G^{\prime}$ is monotone decreasing, then so is $G^{\prime} / G$. But, $G^{\prime \prime}=f$ and 
$G^{\prime}=F$. Therefore if $f / F$, is monotone decreasing, then so is $G^{\prime} / G$. Assertion (ii) follows from this fact and Remark 1.

Corollary 1. If the density function, $f$, is monotone decreasing, then its c.d.f., $F$, and its left side integral, $G$, are both log-concave.

Proof: Since $F$ is a c.d.f., it must be that $F$ is monotone increasing. Therefore if $f$ is monotone decreasing, it must be that $f(x) / F(x)$ is monotone decreasing. But $\left(\frac{f(x)}{F(x)}\right)^{\prime}=(\ln F(x))^{\prime \prime}$. Therefore if $f$ is monotone decreasing, $F$ must be log-concave. Then $G$ must also be log-concave since, according to Theorem $1, \log$ concavity of $F$ implies log-concavity of $G$.

\section{Reliability Theory and Log-concavity of Right Side Integrals.}

Reliability theory is concerned with the time pattern of survival probability of an existing machine or organism. The length of remaining life for an object is modelled as a random variable $\mathbf{X}$ with c.d.f., $F(x)$, and with support $(\ell, h)$. Reliability theory deals with the properties of the "right tail" of the distribution of $\mathbf{X}$. The reliability function of $\mathbf{X}$ is defined to be $\bar{F}$, where $\bar{F}(x)=1-F(x)$, and the failure rate (also known as the hazard function) is defined by $r(x)=f(x) / \bar{F}(x)$. It is also useful to denote the right side integral of the reliability function by $H(x)=\int_{x}^{h} \bar{F}(t) d t$. The following result, concerning right side log-concavity is dual to Theorem 1.

\section{Theorem 2.}

i. If the density function, $f$, is $\log$ concave on $(\ell, h)$, then the reliability function, $\bar{F}$, is also log-concave on $(\ell, h)$.

ii. If the reliability function, $\bar{F}$, is log-concave on $(\ell, h)$, then the right hand integral, $H(x)$, is a log-concave function on on $(\ell, h)$.

Proof: To prove Assertion (i), apply Lemma 1 to the function $\bar{F}(x)=1-F(x)$. Since $F$ is a cumulative distribution function, it must be that $\bar{F}(h)=0$ and $\bar{F}$ is a monotone decreasing function. Therefore if $\bar{F}^{\prime \prime}(x) / \bar{F}^{\prime}(x)$ is a decreasing function, then so is $\bar{F}^{\prime}(x) / \bar{F}(x)$. But $\bar{F}^{\prime}(x)=-f(x)$ and $\bar{F}^{\prime \prime}(x)=-f^{\prime}(x)$ for all $x \in[a, b]$. Therefore, $\bar{F}^{\prime \prime}(x) / \bar{F}^{\prime}(x)=f^{\prime}(x) / f(x)$. It follows that if $f^{\prime}(x) / f(x)$ is monotone decreasing, then $\bar{F}^{\prime}(x) / \bar{F}(x)$ is monotone decreasing. Therefore if $f$ is log-concave, then $\bar{F}$ must be log-concave.

To prove Assertion (ii), apply Lemma 1 to the function $H(x)$. Evidently, $H(h)=0$ and $H(x)$ is monotone decreasing in $x$. From Lemma 1 , it follows that if $H^{\prime \prime}(x) / H^{\prime}(x)$ is monotone decreasing, then so will be $H^{\prime}(x) / H(x)$. In this case, $H^{\prime}(x)=-\bar{F}(x)$ and $H^{\prime \prime}(x)=f(x)$. Therefore if $H^{\prime \prime}(x) / H^{\prime}(x)=-f(x) / F(x)$ is monotone decreasing, then $H^{\prime}(x) / H(x)=-\bar{F}(x) / H(x)$ must also be monotone decreasing. It follows that if $\bar{F}$ is log-concave, then $H$ is log-concave.

Since the failure rate is defined by $r(x)=f(x) / \bar{F}(x)=-\bar{F}(x)^{\prime} / \bar{F}(x)$, it must be that the reliability function $\bar{F}$ is log-concave if and only if the failure rate is monotone increasing in $x$. From this fact and from Theorem 2, the following is immediate.

Corollary 2. If the density function, $f$, is log-concave on $(a, b)$, then the failure rate is monotone increasing on $(a, b)$. If the failure rate is monotone increasing on $(a, b)$, then $H^{\prime}(x) / H(x)$ is monotone decreasing.

Corollary 3. If the density function $f$ is monotone increasing, then the reliability function, $\bar{F}$, is log-concave.

Proof: Since $\bar{F}$ is a reliability function, it must be that $\bar{F}$ is monotone decreasing. Therefore if $f$ is monotone increasing, the failure rate $f / \bar{F}$ must be monotone increasing. But increasing failure rate is equivalent to a $\log$-concave reliability function. 


\section{Applications}

\section{A "Lemons Market" with Costly Appraisals.}

Consider a population of objects of varying quality all of which must be sold by their current owners. The current owner of each object knows its quality, but buyers know only the distribution function of quality in the population. To a potential buyer of an unappraised object, the quality of an object is a random variable random variable $\mathbf{X}$, which will be revealed to him only after he purchases the object. An object known to be of quality, $x$, will command a price of $\$ x$. At some cost, the owner of any object can have it credibly and accurately appraised. Here we sketch a few results that are developed more thoroughly in another paper, Bagnoli and Bergstrom (1989b).

In equilibrium there will be some pivotal quality, $x^{*}$, such that the owners of objects of quality $x>x^{*}$ will have their objects appraised and sell them for $x$. Objects worse than $x^{*}$ will not be appraised and will sell for the average value of objects that are no better than $x^{*}$. The owner of the object of pivotal quality will be indifferent between appraising and not appraising her object. Let the cost of having an object appraised be $c$. Then if the owner of an object of quality $x^{*}$ has it appraised, she will receive a net revenue of $x^{*}-c$. If she does not have her object appraised, it will sell for the average value of objects that are not appraised. This is the set of objects which are no better than $x^{*}$. Let us define the function $\delta(x)$, where $\delta(x)$ is the difference between $x$ and the average quality of objects that are no better than $x .^{1}$ If $c$ is the cost of an appraisal, then the owner of the pivotal object will be indifferent between appraising and not appraising if $\delta\left(x^{*}\right)=c$. Evidently, equilibrium will be unique if $\delta(x)$ is a monotone function.

More formally, $\delta(x)$ is the difference between $x$ and the expected value of the random variable obtained by truncating the distribution of $\mathbf{X}$ to the interval $(\ell, x)$.

Definition. For a random variable $\mathbf{X}$, with support $(\ell, h)$, define $\delta(x)=x-\int_{\ell}^{x} t \frac{f(t)}{F(x)} d t$.

If we integrate the definition of $\delta(x)$ by parts, we have $\delta(x)=G(x) / G^{\prime}(x)$, where $G(x)=\int_{\ell, x} F(t) d t$. From Remark 1 it follows that $G(x) / G^{\prime}(x)$ is monotone increasing if and only if $G$ is log-concave. This leads to

Remark 2. The function $\delta(x)$ monotone increasing if and only if $G(x)$ is log concave. ${ }^{2}$

Remark 2, together with Theorem 1 gives us the following conditions for determining whether $\delta(x)$ is a monotone increasing function.

Theorem 3. Either of the following conditions is sufficient for $\delta(x)$ to be monotone increasing.

i. $f(x)$ is log-concave.

ii. $F(x)$ is log-concave.

\section{Mean Residual Lifetime in Reliability Theory}

The mean residual lifetime function evaluated at $x$ is the expected length of remaining life for a machine of age $x$. Thus we define $M R L(x)=\int_{x}^{h} t f(t) d t-x$. If $M R L(x)$ is a monotone decreasing function, then a machine will "age" with the passage of time, in the sense that it's expected remaining lifetime will diminish

1 This notion seems commonplace enough to have a familiar name in ordinary English, but it doesn't appear to have one. We will call it the $\delta$ function.

2 This result is reported and proved in Goldberger(1983). Goldberger attributes his proof to Gary Chamberlin. 
as it gets older. The $M R L$ function is characterized by Muth (1975) and Swartz (1973). Our methods yield a simple proof of the following result. ${ }^{3}$

Theorem 4. Let the random variable $\mathbf{X}$ represent the length of life. Either of the following conditions is sufficient for mean residual lifetime $M R L(x)$ to be a monotone decreasing function.

i. The density function, $f(x)$, is log-concave.

ii. The failure rate, $r(x)$, is a monotone increasing function.

Proof: Integrating $M R L(x)$ by parts, one finds that $M R L(x)=\int_{x}^{h} \bar{F}(\xi) d \xi / \bar{F}(x)$. Let $H(x)=\int_{x}^{h} \bar{F}(\xi) d \xi$. Then $M R L(x)=H(x) / H^{\prime}(x)$, so that $M R L(x)$ is a decreasing function if and only if $H(x)$ is log-convex. According to Theorem 2(ii), $H(x)$ will be log-convex if $r(x)$ is an increasing function. This proves the sufficiency of Condition (ii). According to Theorem 2(i), log-concavity of $f(x)$ implies that $r(x)$ is monotone increasing, which in turn implies that $M R L(x)$ is monotone decreasing. This proves the sufficiency of Condition (i)

\section{Transformations, Truncations, and Mirror Images.}

\section{Transformations}

The log-normal distribution is a familiar example of a distribution function that is defined by a transformation of variables. Specifically the $\log$-normal distribution is defined on $(0, \infty)$ so that its c.d.f. satisfies $F(x)=$ $N(\ln (x))$ where $N$ is the c.d.f. of the normal distribution. As we will show, the normal distribution has a $\log$-concave density function. The transformation function $\ln (x)$ is evidently a monotone increasing concave function. As it turns out, these two facts are sufficient to imply that the c.d.f. of the log normal distribution is log-concave. On the other hand, the density function of the log-normal distribution is not log-concave.

The following results help to clarify the issue of inheritance of log-concavity under concave transformations of variables.

Lemma 2. Let $t$ be a strictly increasing function, defined on the real interval $(a, b)$, and let $g$ be a function with domain $(t(a), t(b))$. Define the function $\hat{g}$ on $(a, b)$ so that $\hat{g}(x)=g(t(x))$ for all $x \in(a, b)$. If $t$ is concave and twice differentiable and if $g$ is log-concave on $(t(a), t(b))$, then $\hat{g}$ is log-concave on $(a, b)$.

Proof: Calculating derivatives, one finds that $(\ln g(x))^{\prime \prime}$ is of the same sign as $\frac{g^{\prime \prime}(x)}{g^{\prime}(x)}-\frac{g^{\prime}(x)}{g(x)}$. Similar calculations show that $(\ln \hat{g}(x))^{\prime \prime}$ is of the same sign as $\frac{g^{\prime \prime}(x)}{g^{\prime}(x)}+\frac{t^{\prime \prime}(x)}{t^{\prime}(x)}-\frac{g^{\prime}(x)}{g(x)}$. Since $t$ is monotone increasing and concave, it must be that $\frac{t^{\prime \prime}(x)}{t^{\prime}(x)}<0$. Therefore if $g$ is log-concave, then $\frac{g^{\prime \prime}(x)}{g^{\prime}(x)}+\frac{t^{\prime \prime}(x)}{t^{\prime}(x)}-\frac{g^{\prime}(x)}{g(x)}<0$, which implies that $\hat{g}$ is $\log$-concave.

Lemma 3. Let $t(x)=a x+b$ where $a$ may be either positive or negative. Then if $\hat{g}(x)=g(t(x))$ for all $x$, then $\hat{g}$ is log-concave if and only if $g$ is log-concave.

Proof: The result follows immediately from the fact that $(\ln \hat{g}(x))^{\prime \prime}=a^{2}(\ln g(x))^{\prime \prime}$.

Theorem 5. Let the random variable $\mathbf{X}$ have distribution function $F$ and let $\hat{\mathbf{X}}$ have distribution function $\hat{F}$, where $\hat{F}(x)=F(t(x))$.

i. If $F$ is log-concave and $t(x)$ is twice differentiable, strictly increasing, and concave, then $\hat{F}$ is log-concave.

3 This result has also been proved by Muth (1975). 
ii. Let $f$ and $\hat{f}$ be the density functions for $\mathbf{X}$ and $\hat{\mathbf{X}}$, respectively. If $f$ is log-concave and $t$ is linear then $\hat{f}$ is log-concave.

Proof: Result (i) immediate from Lemma 2. For Result (ii), notice that $\hat{f}(x)=f(t(x)) t^{\prime}(x)$. If $t(x)=a x+b$, then $(\ln f(t(x)))^{\prime \prime}=a^{2}(\ln f(t))^{\prime \prime}$. Therefore $\hat{f}$ is log-concave if and only if $f$ is log-concave.

When we discuss the log-normal distribution in detail, we will see that the log normal distribution is an example that demonstrates the following fact.

Remark. Log-concavity of $f$ and concavity of $t$ are not sufficient to imply log-concavity of $\hat{f}$.

Mirror-image Transformations.

Of special interest for our theory is the transformation of random variables into their "mirror-images". Let $\mathbf{X}$ be a random variable with cumulative distribution function $F$ and support $(\ell, h)$. The mirror-image random variable is a random variable $\mathbf{X}^{*}$ with support $(-h,-\ell)$ whose cumulative distribution function is $F^{*}$, where $F^{*}(x)=\bar{F}(-x)=1-F(-x)$. Then

Theorem 6. If $\mathbf{X}$ and $\mathbf{X}^{*}$ are mirror-image random variables, then

$i$ If either of the these two random variables has a log-concave density function, so does the other.

ii One of these two random variables has a log-concave c.d.f. if and only if the other has a log-concave reliability function.

iii One of these random variables has a monotone increasing delta function if and only if the other has a monotone decreasing mean residual lifetime function.

Proof:

If $\mathbf{X}$ and $\mathbf{X}^{*}$ are mirror-image random variables then their c.d.f.'s are related by $F^{*}(x)=\bar{F}(-x)$. Their density functions are related by $f^{*}(x)=f(-x)$ and the left integral, $G$ of the c.d.f. of $\mathbf{X}$ is related to the right integral, $H^{*}$, of the reliability function of $\mathbf{X}^{*}$ by $H^{*}(x)=G(-x)$.

Since $f^{*}(x)=f(-x)$, it follows from Lemma 3 that $f^{*}$ is log-concave if and only if $f$ is log-concave.

Since $F^{*}(x)=\bar{F}(-x)$, it also follows from Lemma 3 that $F^{*}$ is log-concave if and only $F$ is log-concave. Therefore the c.d.f., $F^{*}$ of the mirror-image random variable, $\mathbf{X}^{*}$ will be log-concave if and only if the reliability function, $\bar{F}$ of $\mathbf{X}$ is $\log$-concave.

The delta function is monotone increasing if and only if $G$ is log-concave and the mean residual lifetime is monotone decreasing if and only if $H$ is log-concave. Since $H^{*}(x)=G(-x)$, it follows from Lemma 3 that $H^{*}$ is log-concave if and only if $G$ is log-concave. This means that the mirror-image random variable has decreasing mean residual lifetime if and only if the original random variable has an increasing delta function. Since the mirror-image of the mirror-image is the original distribution, it also follows that the mirror-image random variable has an increasing delta function if and only if the original random variable has a decreasing mean residual lifetime.

If a random variable, $\mathbf{X}$, has a density function that is symmetric around zero, then $\mathbf{X}$ will be its own mirror-image. In this case Theorem 6 has the following Corollary.

Corollary 3. If the random variable $\mathbf{X}$ has a distribution that is symmetric around zero, then

$i$ The c.d.f. will be log-concave if and only if the reliability function is log-concave.

ii The delta function will be monotone increasing if and only if the mean residual lifetime is monotone decreasing. 


\section{Truncations}

Suppose that a random variable $\mathbf{X}$ with domain $[\ell, h]$ is "truncated" to form a new random variable $\mathbf{X}^{*}$, where the probability mass is restricted to a subinterval, $\left[\ell^{*}, h^{*}\right]$, of $[\ell, h]$ while the relative probability density of any two points in this subinterval is unchanged. If $F$ is the c.d.f. of $\mathbf{X}$ and $F^{*}$ is the density

function of $\mathbf{X}^{*}$, it must be that $F^{*}(x)=\frac{F(x)-F\left(\ell^{*}\right)}{F\left(h^{*}\right)-F\left(\ell^{*}\right)}$. But this means that the c.d.f. of $\mathbf{X}$ is just a linear transformation of the c.d.f. of $\mathbf{X}^{*}$. It follows that the density functions of the two random variables are also linear transformations of each other, as are the left and right side integrals of the c.d.f.'s. Clearly if a function is log-concave, so will be any linear transformation of that function. Therefore we can claim the following.

Theorem 7. If $\mathbf{X}$ is a random variable with support $[\ell, h]$ and if $\mathbf{X}^{*}$ is a truncation of $\mathbf{X}$, then the density function of $\mathbf{X}^{*}$ is log-concave if the density function of $\mathbf{X}$ is $\log$-concave. Furthermore, log-concavity of the c.d.f. and of its left and right integrals are inherited under truncation.

\section{Log-concavity Properties of Some Common Distributions.}

This section is a catalog of information about the log-concavity of density functions, distribution functions, reliability functions, and of the integrals of the distribution functions and reliability functions. Extensive discussions of these distributions can be found in Patel (1976), and Johnson and Kotz (1970). Neither book deals extensively with log-concavity. Patel reports known results on whether the distributions that are most commonly studied by reliability theorists have increasing or decreasing failure rates and mean residual lifetime functions.

The easiest distributions to deal with are the ones with log-concave density functions, $f$. Then according to Theorems 1 and 2, the left side integrals, $F$ and $G$, and the right side integrals, $\bar{F}$ and $H$, will be logconcave functions. From these theorems, it follows that if $f$ is log-concave, then the $\delta$ function is monotone increasing, the failure rate is monotone increasing, and the mean residual lifetime function is monotone decreasing.

Families of distributions that always have log-concave density functions include the uniform distribution, the normal distribution, the logistic distribution, the extreme-value distribution, the chi-square distribution, the chi distribution, the exponential distribution, and the Laplace distribution. Some families of distributions have log-concave density functions for certain parameter values and not for others. Such families include the gamma distribution, the beta distribution, the Weibull distribution, and the power function distribution. The Student's $t$ distribution, the Cauchy distribution, the $F$ distribution, the Pareto distribution and the log-normal distribution do not have log-concave density functions for any parameter values.

If a distribution function does not have log-concave density, it still may be that the c.d.f., $F$, is logconcave or the reliability function, $\bar{F}$, is log-concave. For the distributions listed below, we present all of the information that we have been able to discover about log concavity of the density function and its integrals.

We state our results for "standardized forms" of random variables, where the scale and "zero" of the random variable is chosen for simplicity of expression. According to Theorem 5, the log-concavity properties are all preserved under linear transformations, so that these theorems apply to the family of distributions obtained by performing linear transformations on the random variable. For example, we discuss the standard normal distribution, with mean 1 and standard deviation zero. But our characterization applies to all univariate normal distributions, since any normally distributed random variable is a linear transformation of a random variable with a standard normal distribution.

Whatever we learn about log-concavity of distributions applies immediately to truncations of these distributions, since log-concavity of a density function or of its integrals is inherited under truncation. It is standard practice for reliability theorists to concern themselves only with distributions that are bounded from below by zero. It may therefore seem surprising that we apply the definitions of reliability theory to distributions whose support may be unbounded from below. Aside from considerations of mathematical symmetry, there is a good practical reason for doing so. As we remarked, the log-concavity properties that 
we study are preserved under truncations of random variables. Therefore if a distribution with unbounded support is log-concave, so will be every truncation of that distribution. If we find that a distribution has, for example, a log-concave reliability function with a support that is unbounded from below, then we know that the distribution could be truncated to any bounded interval and employed as an example with log-concave reliability.

\section{The Uniform Distribution}

The uniform distribution, defined on the interval $[0,1]$ has density $f(x)=1$. For this function there are simple expressions for the c.d.f, the $\delta$ function, the failure rate, and the mean residual lifetime. The cumulative distribution function is $F(x)=x$. The delta function is defined by $\delta(x)=\int_{0}^{x} F(t) d t / F(x)=x / 2$. The failure rate is $r(x)=f(x) / \bar{F}(x)=\frac{1}{1-x}$ and the mean residual lifetime is $M R L(x)=\int_{x}^{1} \bar{F}(t) d t / \bar{F}(t)=$ $(1-x) / 2$. One sees immediately that the density function is (weakly) log-concave, that the delta function is monotone increasing, the failure rate is monotone increasing, and the mean residual lifetime is monotone decreasing. As we know from Theorems 1 and 2, each of these latter properties must follow from the log-concavity of $f$.

\section{The Normal Distribution.}

Since the normal cumulative distribution function does not have a closed form, it is quite difficult to determine directly whether the c.d.f. is log-concave or to determine whether the $\delta$ function, the failure rate, and the mean residual lifetime function are monotonic. But using the inheritance theorems, each of these properties is easily established.

The standard normal probability distribution has probability density

$$
f(x)=\frac{1}{\sqrt{2 \pi}} e^{-x^{2} / 2} .
$$

Then $(\ln f(x))^{\prime}=-x$ and $(\ln f(x))^{\prime \prime}=-1<0$. Therefore the normal distribution has log-concave density. The remaining properties follow as consequences of Theorems 1 and 2 .

\section{The Logistic Distribution.}

The logistic distribution has cumulative distribution function $F(x)=\frac{1}{1+e^{-x}}$ and density $f(x)=\frac{e^{-x}}{\left(1+e^{-x}\right)^{2}}$. Calculation shows that $(\ln f(x))^{\prime}=-1+2(1-F(x))$ and $(\ln f(x))^{\prime \prime}=-2 f(x)<0$, which implies that the logistic distribution has log-concave density.

\section{The Extreme-Value Distribution.}

The extreme value distribution has density function $f(x)=\exp \left\{-e^{-x}\right\}$. In this case, $(\ln f(x))^{\prime \prime}=-e^{-x}<0$, which means that this distribution has log-concave density. The extreme value distribution arises as the limit as $n \rightarrow \infty$ of the greatest value among $n$ independent random variables.

\section{The Exponential Distribution}

The exponential distribution has support, $[0, \infty]$, and density function, $f(x)=\lambda e^{-\lambda x}$. The c.d.f. is $F(x)=$ $1-e^{-\lambda x}$. The density function is log-linear and hence $\log$-concave, with $(\ln f(x))^{\prime \prime}=0$, so $f(x)$ i. Since $f^{\prime}(x)<0$ and $F^{\prime}(x)>0$ for all $x$, it must be that $f(x) / F(x)$ is monotone decreasing and hence $F$ is strictly $\log$-concave. From Theorem 2, it follows that $\delta(x)$ is a monotone increasing function.

As Barlow and Proschan (1975) point out, the exponential distribution is the only distribution for which the failure rate and the mean residual lifetime is constant. The failure rate, $f(x) / \bar{F}(x)=\lambda$. The mean 
residual lifetime function is $M R L(x)=\int_{x}^{h} \bar{F}(t) d t / \bar{F}(x)=1 / \lambda$. If the lifetime of an object has an exponential distribution, then it does not "wear out" over time. That is to say, the probability of failure and the expected remaining lifetime remain constant so long as the object "survives".

\section{The Laplace Distribution}

The Laplace distribution has density function $f(x)=\frac{\lambda}{2} e^{-\lambda|x|}$ where $\lambda>0$. The c.d.f. is

$$
F(x)= \begin{cases}\frac{1}{2} e^{\lambda x}, & \text { if } x \leq 0 \\ 1-\frac{1}{2} e^{-\lambda x}, & \text { if } x \geq 0\end{cases}
$$

The Laplace density function is sometimes known as the "double exponential" distribution, since it is proportional to the exponential density for positive $x$ and to the mirror-image of the exponential distribution for negative $x$. For the Laplace distribution, $\ln f(x)=-\lambda|x|$. The function $-\lambda|x|$ is clearly a concave function, although its derivative does not exist at $x=0$.

\section{The Weibull Distribution.}

The Weibull distribution with parameter $c>0$ has density function

$$
f(x)=c x^{c-1} e^{-x^{c}}
$$

defined for $x \in(0, \infty)$. Calculation shows that $(\ln f(x))^{\prime \prime}=(1-c) x^{-2}\left(1+c x^{c}\right)$. This expression is negative for $c>1$, zero for $c=1$ and positive for $c<1$. Therefore the density function is (strictly) log-concave if $0<c<1, \log$-linear if $c=1$, and $\log$-convex if $c>1$.

If $0<c<1$, then calculation shows that $f^{\prime}(x)<0$ for all $x>0$. From Corollary 1 it follows that the c.d.f. is log-concave and the delta function is monotone increasing.

For the Weibull distribution, the reliability function is $\bar{F}(x)=1-F(x)=e^{-x^{c}}$. Then $(\ln \bar{F}(x))^{\prime \prime}=$ $-c(c-1) x^{c-2}$ is positive if $c<1$ and nonpositive if $c \geq 1$. So the reliability function is log-concave when $c \geq 1$ and $\log$-convex when $c<1$. It follows that for the Weibull distribution with $0<c<1$, the failure rate is a decreasing function of age.

\section{The Power Function Distribution.}

The power function distribution has c.d.f, $F(x)=x^{\beta}$, with support on the interval, $(0,1)$. The density function is $f(x)=\beta x^{\beta-1}$. Calculation shows that $(\ln f(x))^{\prime \prime}=\frac{1-\beta}{x^{2}}$, so that the density function is logconcave if $\beta \geq 1$ and log-convex if $0<\beta<1$.

The power function has a log-concave c.d.f. for all positive $\beta$. This is the case since $(\ln F(x))^{\prime \prime}=$ $\frac{-\beta}{x^{2}}<0$. For the power function distribution, we can actually calculate the delta function, which is $\delta(x)=$ $\int_{\ell}^{x} F(t) d t / F(x)=x /(\beta+1)$. Evidently, $\delta(x)$ is monotone increasing for all $\beta>0$. This is as it must be, since log-concavity of $F(x)$ implies that $\delta(x)$ is monotone increasing.

The reliability function is $\bar{F}(x)=1-x^{\beta}$. With calculations, one finds that

$$
(\ln \bar{F}(x))^{\prime \prime}=\frac{\beta x^{\beta-2}\left(1-\beta-x^{\beta}\right)}{\left(1-x^{\beta}\right)^{2}} .
$$

is of the same sign as $1-\beta-x^{\beta}$. This expression is positive for $x$ near 0 and negative for $x$ near 1 . Therefore, the reliability function, is neither log-concave nor log-convex on $(0,1)$. For this distribution, the right side integral of the reliability function is $H(x)=\frac{\beta-x \beta+1}{1+\beta}-x$. Computations show that this function is neither log-concave nor log-convex. 


\section{The Gamma Distribution}

The gamma distribution has density function

$$
f(x)=\frac{x^{m-1} \theta^{m} e^{-x \theta}}{\Gamma(m)}
$$

defined for $x \in(0, \infty)$, where $\theta>0$ and $m>0$. Calculation shows that $(\ln f(x))^{\prime \prime}=\frac{1-m}{x^{2}}$. Therefore the density function is strictly log-concave if $m>1$.

For the gamma distribution with $m<1, f$ is strictly log-convex. But in this case, $f^{\prime}(x)<0$ for all $x>0$. Therefore, according to Corollary 1, the c.d.f. is log-concave and from Theorem 1 it follows that the left side integral of the c.d.f. is log-concave.

Barlow and Proschan (1981, page 75) prove that if $m<1$, then the failure rate is a monotone decreasing function of age. This property is equivalent to log-convexity of the reliability function.

\section{The Chi-squared Distribution.}

The chi-square distribution with $n$ degrees of freedom is a gamma distribution with $\theta=2$ and $m=n / 2$. Recall that the sum of the squares of $n$ independent standard normal random variables has a chi-square distribution with $n$ degrees of freedom. Since the gamma distribution has a log-concave density function for $m \geq 1$, we can conclude that the sum of the squares of two or more independent standard normal random variables has a log-concave density function.

\section{The Chi Distribution}

The chi distribution has support $\{x \mid x>0\}$ and density function,

$$
f(x)=\frac{x^{(n / 2)-1} e^{-n / 2} x^{2}}{2^{n / 2} \Gamma(n / 2)}
$$

where $n$ is a positive integer. If a random variable $\mathbf{X}$ has a chi-square distribution with $n$ degrees of freedom, then $\sqrt{\mathbf{X} / n}$ has a chi distribution parameter with parameter $n$. The sample standard deviation from of the sum of $n$ independent standard normal variables has a chi distribution. The chi distribution with $n=2$ is sometimes known as the Rayleigh distribution amd the chi distribution with $n=3$ is sometimes known as the Maxwell distribution.

For this distribution, $(\ln f(x))^{\prime \prime}=-\frac{n-1}{x^{2}}-n<0$. Therefore the chi distribution has a log-concave density function.

\section{The Beta Distribution}

The beta distribution has density function

$$
f(x)=\frac{x^{a-1}(1-x)^{b-1}}{B(a, b)}
$$

defined for $x \in(0,1)$, where $a>0$ and $b>0$. Calculation shows that $(\ln f(x))^{\prime \prime}=\frac{1-a}{x}+\frac{1-b}{x}$. Therefore if $a \geq 1$ and $b \geq 1$, then the density function is log-concave. If $a<1$ and $b<1$, then the density function is $\log$-convex. If $a<1$ and $b>1$ or if $a>1$ and $b<1$, then the density function is neither log-convex nor log-concave on $(0,1)$.

We do not have general results on the log-concavity of the c.d.f and reliability function for beta distributions where $a>1$ and $b<1$ or where $a<1$ and $b>1$. We have studied two special cases. One is the case of 
$a=.5, b=.5$. The resulting distribution is known as the arc sin distribution. Closed form expressions can be found for the c.d.f. and reliability function, but they are not pretty. Plotting curves with the computer program, Mathematica, shows that the density function and the reliability function are neither log-concave nor log-convex. So far, our numerical efforts have not decisively established whether the integrals of the c.d.f. and the reliability function are log-concave.

Another case that we have worked out, with the aid of Mathematica, is the beta function with $a=2$, $b=.5$. In this case, there are complicated but manageable closed-form expressions for the c.d.f., the reliability function and their integrals. The c.d.f. and its integral are neither log-concave nor log-convex. The reliability function and its integral are log-convex.

\section{The Pareto Distribution}

The Pareto distribution is defined over the non-negative real numbers and has c. d. f., $F(x)=1-x^{-\beta}$ where $\beta>0$. The density function is $f(x)=\beta x^{-\beta-1}$. Then $(\ln f(x))^{\prime \prime}=\frac{\beta+1}{x^{2}}>0$. Therefore the density function is $\log$-convex rather than log-concave. But notice that $f^{\prime}(x)<0$ for all $x$. According to Corollary 1 , this implies that the c.d.f, $F(x)$, is log-concave.

The Pareto distribution is a simple example of a distribution for which the failure rate and the mean residual lifetime behave "unusually". The reliability function for the Pareto distribution is $\bar{F}(x)=x^{-\beta}$. Then $(\ln \bar{F}(x))^{\prime \prime}=\beta / x^{2}>0$. Therefore the the reliability function is log-convex rather than log-concave, which means that the failure rate is a decreasing function of $x$. Where $H(x)$ is defined as $\int_{x}^{\infty} F(t) d t, H(x)$ converges if and only if $\beta>1$ and in this case, $H(x)=\frac{1}{\beta-1} x^{1-\beta}$. Then $(\ln H(x))^{\prime \prime}=\frac{\beta-1}{x^{2}}>0$. Therefore $H(x)$ is log-convex and the mean residual lifetime is a decreasing function of $x$.

\section{The Log Normal Distribution}

If $\mathbf{Y}$ is a $\log$ normal distribution, then $\mathbf{Y}=e^{\mathbf{X}}$ where $\mathbf{X}$ is a normal distribution. Since the normal distribution has a $\log$-concave c.d.f., it follows from Theorem 4, that the log normal distribution also has a concave c.d.f. From Theorem 3 it follows that for a log normally distributed random variable, $\delta(x)$ is increasing.

Unlike the normal distribution, the log normal distribution does not have a log-concave density function. The log normal density function is

$$
f(x)=\frac{1}{x \sqrt{2 \pi}} e^{-(\ln x)^{2} / 2} .
$$

A bit of calculation shows that

$$
(\ln f(x))^{\prime \prime}=\frac{\ln x}{x^{2}} .
$$

Therefore, $f(x)$ is log-concave on the interval $(0,1)$ and log-convex on the interval $(1, \infty)$.

The failure rate of a log normally distributed random variable is neither monotone increasing nor monotone decreasing. (Patel, et.al. (1976)). Furthermore the mean residual lifetime for the log normal distribution is not monotonic, but is increasing for small values and decreasing for large values of X. (see Muth (1975)). We have not found an anaytic proof of either of these last two propositions. As far as we can tell, they have only been demonstrated by numerical calculation and computer graphics.

\section{Student's t Distribution}

Student's $t$ distribution is defined on the entire real line with density function

$$
f(x)=\frac{\left(1+\frac{x^{2}}{n}\right)^{-n+1 / 2}}{\sqrt{n} B(.5, n / 2)}
$$


where $B(a, b)$ is the incomplete beta function and $n$ is referred to as the number of degrees of freedom. For the $t$ distribution $(\ln f(x))^{\prime \prime}=-(n+1) \frac{n-x^{2}}{\left(n+x^{2}\right)^{2}}$. Therefore the density function of the $t$ distribution is log-concave on the central interval $[-\sqrt{n}, \sqrt{n}]$ and log-convex on each of the outer intervals, $[-\infty,-\sqrt{n}]$ and $[\sqrt{n}, \infty]$. although the $t$ distribution itself is not log-concave, a truncated $t$ distribution, where the truncation is restricted to a subset of the interval $[-\sqrt{n}, \sqrt{n}]$ will be log-concave.

We do not have a general, analytic proof of the concavity or non-concavity of the c.d.f. of the $t$ distribution. But numerical calculations (with the program Gauss) show that the c.d.f is neither log-concave nor log-convex for the cases of $n=1,2,3,4$, and 24. Since the $t$ distribution is symmetric, the log reliability function is the mirror-image of the $\log$ of the log c.d.f. Therefore if the c.d.f. is neither log-concave nor log-convex, the reliability function must also be neither concave nor convex.

\section{The Cauchy Distribution.}

The Cauchy distribution is a Student's $t$ distribution with 1 degree of freedom. It is equal to the distribution of the ratio of two independent standard normal random variables.

The Cauchy distribution has density function $f(x)=\frac{1}{\pi\left(1+x^{2}\right)}$ and c.d.f $F(x)=1 / 2+\frac{\tan ^{-1}(x)}{\pi}$. Then $(\ln f(x))^{\prime \prime}=-2 \frac{x^{2}-1}{\left(x^{2}+1\right)^{2}}$. This expression is negative if $|x|<1$ and positive if $|x|>1$. Like the rest of the family of $t$ distributions, the density function of the Cauchy distribution is neither log-concave, nor log-convex.

The integral $\int_{\infty}^{x} F(t) d t$ does not converge for the Cauchy distribution, and therefore the function $G$ is not well-defined.

\section{The F Distribution}

The support of the $F$ distribution is the set of positive real numbers. The $F$ distribution has two integervalued parameters $m_{1}$ and $m_{2}$, known as "degrees of freedom". The density function of an $F$ distribution with $m_{1}$ and $m_{2}$ degrees of freedom is

$$
f(x)=c x^{\left(m_{1} / 2\right)-1}\left(1+\left(m_{1} / m_{2}\right) x\right)^{-\left(m_{1}+m_{2}\right) / 2}
$$

where $c$ is a constant that depends only on $m_{1}$ and $m_{2}$. The $F$ distribution arises in statistical applications as the distribution of the ratio of two independent chi-square distributions with $m_{1}$ and $m_{2}$ degrees of freedom.

For the $F$ distribution,

$$
(\ln f(x))^{\prime \prime}=-\left(m_{1} / 2-1\right) / x^{2}+\left(m_{1} / m_{2}\right)^{2}\left(m_{1}+m_{2}\right) / 2\left(1+m_{1} / m_{2} x\right)^{-2} .
$$

If $m_{1} \leq 2$, then the $F$ distribution is $\log$-convex. For $m_{1}>2$, calculation shows that $(\ln f(x))^{\prime \prime}$ is positive or negative depending on whether $x$ is greater than or less than

$$
\frac{m_{2} \sqrt{\frac{m_{1}-2}{m_{1}+m_{2}}}}{1-\sqrt{\frac{m_{1}-2}{m_{1}+m_{2}}}} .
$$

Therefore the $F$ distribution is neither log-concave nor log-convex. 
So far, we have not produced an example of a distribution that does not have a monotone increasing delta function. Indeed, we have not come across a "named" distribution that has this property. But we do have distributions with increasing mean residual lifetime. According to Theorem 6 , the mirror-image of such a distribution must have a decreasing delta function.

The mirror-image of the Pareto distribution is a particularly convenient example. The mirror-image of the Pareto distribution has support $(-\infty,-1)$ and c.d.f. $F(x)=(-x)^{-\beta}$ where $\beta>0$. For $\beta>1$, $G(x)=\int_{-\infty}^{x} F(t) d t$ converges and $G(x)=(\beta-1)^{-1}(-x)^{1-\beta}$. Then $\delta(x)=G(x) / G^{\prime}(x)=G(x) / F(x)=\frac{x}{1-\beta}$ and $\delta^{\prime}(x)=\frac{1}{1-\beta}<0$.

\section{Mirror-image of the Log Normal Distribution}

The mirror-image Pareto distribution gave us an example of a distribution with a monotone decreasing $\delta$ function. The mirror-image log normal distribution gives us an example of a distribution where the $\delta$ function is decreasing for some some values of $x$ and increasing for others.

The support of the mirror-image log-normal distribution is the set of negative real numbers. Unlike the case of the mirror-image Pareto distribution, we cannot calculate closed form expressions for the c.d.f. or for the $\delta$ function. But we know that both the c.d.f. and the $\delta$ function are non-monotonic because we know that the failure rate and the mean residual lifetime function of the log normal are non monotonic and can apply Theorem 6 . 
Log-concavity of Some Common Distributions

\begin{tabular}{|c|c|c|c|c|c|}
\hline Distribution & Density & c.d.f. & Int of c.d.f & Reliability & Int of Reliability \\
\hline Uniform & log-concave & log-concave & log-concave & log-concave & log-concave \\
\hline Normal & log-concave & log-concave & log-concave & log-concave & log-concave \\
\hline Logistic & log-concave & log-concave & log-concave & log-concave & log-concave \\
\hline Extreme Value & log-concave & log-concave & log-concave & log-concave & log-concave \\
\hline Chi-Squared & log-concave & log-concave & log-concave & log-concave & log-concave \\
\hline Chi & log-concave & log-concave & log-concave & log-concave & log-concave \\
\hline Exponential & log-concave & log-concave & log-concave & log-concave & log-concave \\
\hline Laplace & log-concave & log-concave & log-concave & log-concave & log-concave \\
\hline Weibull $(c \geq 1)$ & log-concave & log-concave & log-concave & log-concave & log-concave \\
\hline Power Function $(\beta \geq 1)$ & log-concave & log-concave & log-concave & log-concave & log-concave \\
\hline $\operatorname{Gamma}(m \geq 1)$ & log-concave & log- concave & log-concave & log-concave & log-concave \\
\hline Beta $(a \geq 1, b \geq 1)$ & log-concave & log-concave & log-concave & log-concave & log-concave \\
\hline Log Normal & mixed & log-concave & log-concave & $*_{\text {mixed }}$ & $*$ mixed \\
\hline Pareto & log-convex & log-concave & log-concave & log-convex & log-convex \\
\hline Power Function $(\beta<1)$ & log-convex & log-concave & log-concave & mixed & mixed \\
\hline Weibull $(c<1)$ & log-convex & log-concave & log-concave & log-convex & $?$ \\
\hline $\operatorname{Gamma}(m<1)$ & log-convex & log-concave & log-concave & log-convex & $?$ \\
\hline Beta $(a=.5, b=.5)$ & log-convex & mixed* & $?$ & $\operatorname{mixed}^{*}$ & $?$ \\
\hline Beta $(a=2, b=.5)$ & mixed & mixed & mixed & log-convex & log-convex \\
\hline Student's $t$ & mixed & mixed $^{*}$ & $?$ & mixed $^{*}$ & $?$ \\
\hline Cauchy & mixed & mixed* $^{*}$ & undefined & mixed $^{*}$ & undefined \\
\hline $\mathrm{F}$ distribution & mixed & $?$ & $?$ & $?$ & $?$ \\
\hline Mirror-Image Pareto & log-convex & log-convex & log-convex & log-concave & log-concave \\
\hline Mirror-Image Log Normal & mixed & mixed & mixed & log-concave & logconcave \\
\hline
\end{tabular}

* denotes answers found, not by analytic means, but by numerical simulation for particular parameter values. See detailed comments on the particular distribution. 


\section{References}

Bagnoli, M., and T.Bergstrom (1989a) "Courtship as a Waiting Game," University of Michigan Working Paper.

Bagnoli, M., and T. Bergstrom (1989b) "Signalling and Costly Appraisals," University of Michigan Working Paper.

Bagnoli, M., and N. Khanna (1989) "Why Are Buyers Represented by Sellers' Agents When Buying a House?," University of Michigan Working Paper.

Barlow, R. and F. Proschan (1981) Statistical Theory of Reliability and Life Testing. New York: Holt Rinehart and Winston.

Barlow, R. and F. Proschan (1965) Mathematical Theory of Reliability. New York: Wiley.

Baron, D. and D Besanko (1984) "Regulation, Asymmetric Information, and Auditing," Rand Journal of Economics, 15, 447-470.

Caplin, A., and B. Nalebuff (1988) "After Hotelling: Existence of Equilibrium for an Imperfectly Competitive Market," Princeton University Working Paper.

Caplin A. and B. Nalebuff (1989) "Aggregation and Social Choice: A Mean Voter Theorem," Princeton University Working Paper.

Flinn, C. and J. Heckman (1983) "Are Unemployment and Out of the Labor Force Behaviorally Distinct Labor Force States?," Journal of Labor Economics, 1, 28-43.

Goldberger, A. (1983) "Abnormal Selection Bias," in Studies in Econometrics, Time Series, and Multivariate Statistics, ed. Karlin, S., Amemiya, T., Goodman, L.. New York: Academic Press, 67-84.

Jegadeesh, N. and B. Chowdhry (1989) "Optimal Pre-tender Offer Share Acquisition Strategy in Takeovers," UCLA working paper.

Johnson, N., and S. Kotz (1970) Continuous Univariate Distributions-I Distributions in Statistics. New York: Wiley.

Laffont, J. and Tirole, J. (1988) "Dynamics of Incentive Contracts," Econometrica, , .

Lewis, T. and D. Sappington (1988) "Regulating a Monopolist with Unknown Demand," American Economic Review, .

Maskin, E., and J. Riley (1984) "Monopoly with Incomplete Information," Rand Journal, 15, 2, .

Matthews, S. (1987) “Comparing Auctions for Risk-Averse Buyers: A Buyer's Point of View," Econometrica, , .

Muth, E. (1977) "Reliability Models with Positive Memory Derived From the Mean Residual Life Function," in Theory and Applications of Reliability, Vol II, ed. Tsokos, C. and I. Shimi. New York: Academic Press, 401-436.

Myerson, R. and Satterthwaite, M. (1983) "Efficient Mechanisms for Bilateral Trading," Journal of Economic Theory, , .

Patel, J.K., C.H. Kapadia, and D. B. Owen, D.B. (1976) Handbook of Statistical Distributions. New York: Marcel Dekker, Inc..

Prèkopa, A. (1973) "On Logarithmic Concave Measures and Functions," Act. Sci. Math. (Szeged), 34, 335-343.

Riordan, M. and D. Sappington (1989) "Second Sourcing," Rand Journal, 2, .

Rob, R. (1989) "Pollution Claims Settlements under Private Information," Journal of Economic Theory, 47,

Swarz, G. (1973) "The Mean Residual Lifetime Function," IEEE Transactions in Reliability, , 108-109. 